\title{
HEAT CAPACITY AND THERMAL RELAXATION OF BULK HELIUM $\rho 21$ VERY NEAR THE LAMBDA POINT
}

\author{
J. A. Lipa*, D. R. Swanson*, J. A. Nissen*, and T. C. P. Chui ${ }^{+}$ \\ *Department of Physics, Stanford University, Stanford, CA. \\ +Jet Propulsion Laboratory, California Institute of Technology, Pasadena, CA.
}

\begin{abstract}
In October 1992 a low temperature experiment was flown on the Space Shuttle in low Earth orbit. The objective of the mission was to measure the heat capacity and thermal conductivity of helium very close to the lambda point with the smearing effect of gravity removed. We report preliminary results from the experiment, and compare them with related measurements performed on the ground. The sample was a sphere of helium $3.5 \mathrm{~cm}$ in diameter contained within a copper calorimeter of very high thermal conductivity. The calorimeter was attached to a pair of high resolution paramagnetic salt thermometers with noise levels in the $10^{-10} \mathrm{~K}$ range and suspended from a high stability thermal isolation system. During the mission we found that the resolution of the thermometers was degraded somewhat due to the impact of charged particles. This effect limited the useful resolution of the measurements to about two nanokelvins from the lambda point. The results reported here are limited to about ten nanokelvins from the transition.
\end{abstract}

\section{INTRODUCTION}

Since the first high resolution measurements of the heat capacity singularity at the lambda point, this transition has become the most important testing ground for theories of second order phase transitions. The transition is very sharp because of the strain-free nature of the fluid and its relatively low compressibility. With sample heights of the order of millimeters, values of $t$ as small as $10^{-7}$ can be reached on Earth before intrinsic distortion is encountered. Here $t=\left|1-T / T_{\lambda}\right|$ is a dimensionless temperature parameter, and $T_{\lambda}$ is the transition temperature. The ultimate limit in ground based measurements is usually encountered when finite size effects ${ }^{1}$ become appreciable, generally near $t \approx$ $5 \times 10^{-8}$. In space, the lambda transition may be sharp to $t \sim 10^{-12}$ or $50^{2}$, in optimal conditions. Since the early 70's the aim of most high resolution experiments in this region has been to test the renormalization group (RG) formalism developed by Wilson ${ }^{3}$ to predict the singular thermodynamic behavior observed in

Joint " $L+1$ " Science Review for USML-1 and USMP-1 with the Microgravity Measurement Group, September 22-24, 1993, Huntsville, Alabama, USA. 
many systems. In particular, this formalism led to numerical predictions for many 'universal' parameters, that is, system independent quantities, such as the exponents governing the divergence of various thermodynamic variables.

Over the past 18 years we have developed the technology to perform a high resolution heat capacity experiment near the lambda point on the Space Shuttle. High resolution thermometry with a resolution of $\sim 3 \times 10^{-10} \mathrm{~K}$ was developed, along with a thermal control system with a stability of about $10^{-8} \mathrm{~K}$. The calorimeter was optimized for a residual acceleration level of $2 \times 10^{-4} \mathrm{~g}$, and a corresponding resolution at the lambda point of $t \sim 4 \times 10^{-10}$. The acceleration level was chosen as a compromise between the lower dc acceleration normally encountered on the Shuttle, and the somewhat higher ac level. The experiment design allows about two orders of magnitude higher resolution than is possible on Earth, leading to a substantially improved measurement of the heat capacity curve and a better estimate of the exponent $\alpha$ characterizing its divergence at the transition temperature. The thermal conductivity of the helium above the transition can also be derived from the relaxation data associated with the heat capacity measurements.

In this paper we briefly describe the technology that was developed to make the measurements, and discuss preliminary results from the mission, which was flown in October 1992. We also compare the results with extrapolations derived from ground-based data.

\section{APPARATUS}

The basic design of the apparatus is similar to that used for earlier heat capacity measurements 4 on helium samples with small vertical height performed at Stanford. A number of structural changes were made to improve its ability to survive launch into space. In this and the following sections we briefly describe the flight design with emphasis on those features most critical for high resolution work.

The most significant problems one is faced with when designing a high resolution heat capacity experiment are temperature resolution and thermal control. Conventional thermometry, e.g. germanium resistance thermometry, allows a resolution approaching $10^{-7}$ near $T_{\lambda}$ with low power dissipation. Higher resolution would require increasing the power input, which rapidly leads to unacceptable thermal gradients. Since the goal of our experiment was to achieve a resolution of $4 \times 10^{-10}$, we were immediately faced with the development of a new high resolution thermometer (HRT). The device we developed makes use of superconducting technology to achieve a resolution of about $3 \times 10^{-10} \mathrm{~K}$ in a $1 \mathrm{~Hz}$ bandwidth with negligible power dissipation, and is briefly described below.

To make the heat capacity measurements with the desired resolution it is necessary to meter energy into the sample in units as small as $10^{-9} \mathrm{C}$ Joules, where $C$ is the heat capacity of the sample. In our case, for a heater that operates for a few seconds, this corresponds to powers of the order of $10^{-8} \mathrm{~W}$. 
In addition, during the period used to measure the corresponding temperature rise, the variability of the background power input must be less than $\sim 10^{-10} \mathrm{~W}$. This means that very careful thermal control of the sample environment is necessary. To achieve this, we built a four stage thermal control system which used a HRT on the inner stage as a fine control sensor. This control system was the major portion of the low temperature apparatus which comprised the flight instrument. It is also described below. A third item of great importance is a helium dewar capable of operating in space. We made use of the previously flown JPL low temperature facility which has the capability of easily operating near $2 \mathrm{~K}$ in zero gravity. The performance of this system is described elsewhere ${ }^{5}$.

\section{THERMOMETRY}

The construction of the HRTs has been described in some detail elsewhere ${ }^{6}$. Briefly, they consist of a superconducting tube surrounding a crystal of paramagnetic salt which is thermally coupled to the experiment. The tube applies a fixed magnetic field to the salt. As the temperature of the experiment changes, so does the magnetization of the salt, due to its Curie law susceptibility. A superconducting pickup loop is wound on the salt and couples the changes in magnetization to an if SQUID magnetometer for readout. A schematic view of the device is shown in figure 1 . The HRTs were designed to be mechanically very rugged, and all critical wiring was bonded as firmly as possible. The salt pill and pickup coil assembly were heavily coated with a vacuum grease/kerosene mixture which froze early in the cool-down cycle. The holder of this assembly was in turn locked into the flux tube by differential contraction.

To reduce the effect of the varying orientation of the HRTs with respect to the Earth's magnetic field, three layers of shielding were used. The HRT flux tube itself has an extremely high theoretical shielding factor at the location of the pickup loop, but tests showed ${ }^{7}$ that this could be compromised by flux creep through the walls. The maximum safe shielding factor from this tube was estimated to be about $5 \times 10^{9}$. A second superconducting shield was added around the instrument vacuum can with a theoretical shielding factor of about 200 . Also, a layer of moly-permalloy was placed around the outside of the dewar to gain another factor of 100 . Tests of the complete assembly showed no detectable signal for fields of the order of $1 \mathrm{G}$ applied externally.

For a field of $100 \mathrm{G}$ trapped in the flux tube, the sensitivity of a HRT is typically in the range $3-4$ $\Phi_{0} / \mu \mathrm{K}$, where $\Phi_{0}$ is the quantum of magnetic flux. Since the SQUID noise level is at least as low as $10^{-4}$ $\Phi_{0} / \sqrt{H z}$, a resolution of $\sim 3 \times 10^{-11} \mathrm{deg}$ is possible in principle. In general, a much larger noise level is seen. The noise characteristics of a HRT can be studied by attaching it to a large thermal reservoir and reducing the heating rate to below $10^{-12} \mathrm{~K} / \mathrm{sec}$ by careful thermal control. A typical noise spectrum obtained this way is shown in figure 2 . The noise data agrees well with the prediction of the fluctuation- 
dissipation theorem applied to temperature fluctuations ${ }^{8}$. This is shown by the solid line in the figure, with no adjustable parameters. The HRT noise per unit bandwidth in the low frequency region is normally about $3 \times 10^{-10} \mathrm{~K} V \mathrm{~Hz}$. The absolute drift rate of the HRTs has been found to be $\sim 10^{-14} \mathrm{~K} / \mathrm{sec}$ by observing the apparent location of the lambda transition as a function of time in various high resolution experiments.

\section{THERMAL CONTROL SYSTEM}

The thermal control system used in the flight experiment consisted of a multi-stage thermal platform located in the helium dewar, and an electronics assembly that housed the servos for the various levels of thermal control, along with the readout electronics for the HRTs. It has been described in some detail elsewhere ${ }^{9}$. The platform (shown in figure 3) consists of a vacuum shell about $20 \mathrm{~cm}$ in diameter and $60 \mathrm{~cm}$ long surrounding four thermal control stages in series and the calorimeter. A set of four HRTs are housed in the lower part of the assembly and are surrounded by a thermal shield attached to the innermost stage of the control system. A solenoid is mounted on the outside of the lower portion of the vacuum can to allow the application of a magnetic field to the HRTs during initial cool-down. A cylindrical superconducting shield open at one end surrounds the assembly to minimize the effects of external fields on the HRTs.

A tripod structure with legs of stainless steel tubing hangs from the lid of the vacuum shell. Three OFHC copper rings intersect the legs of the tripod at intervals of about $3 \mathrm{~cm}$. These rings form the first three stages of the isolation system and also serve to stiffen the tripod. Each stage has a germanium resistance thermometer (GRT) and heater for temperature readout and servo control. The thermal isolation of these stages is sufficient to reduce the thermal inhomogeneities to the point where they are dominated by the dissipation in the heater on stage 3 . This effect is then limited by reducing the offset between the operating temperatures of stages 2 and 3 to the minimum possible value. Stage 4 was equipped with two HRTs, GRTs and heaters. Thermal control of this stage had two modes: a coarse mode with the GRT as sensor, and a fine mode with the HRT as sensor. Gradients in this stage were again minimized by reducing the temperature offset to stage 3 .

The calorimeter assembly, stage 5 , consisted of a spherical sample container, a superfluid tight valve, and pairs of HRTs, GRTs and heaters. The configuration was designed to minimize temperature offsets between the HRTs and the sample container. This was done by providing large area contacts between the HRTs and the container, thermally separated from the tripod attach point. Thermal gradient control was improved by deactivating the GRTs during high resolution measurements and using the heater only when step temperature changes were desired. In operation, temperature stability of stage 5 was obtained by fine adjustment of the temperature set point of stage 4 . The limiting factor on thermal 
control of stage 5 appeared to be variations in stray power pickup in the circuits attached to it, and charged particle dissipation in the structure. The sample container was a copper sphere of $3.5 \mathrm{~cm}$ internal diameter and $2 \mathrm{~mm}$ minimum wall thickness. The copper ${ }^{10}$ had a residual resistance ratio of over 4000 . The sample container fill valve was held closed by a spring and opened by applying $\sim 450 \mathrm{psi}$ to a bellows system attached to the stem. The valve seal was formed between a beryllium-copper knife edge and a gold-plated copper flat. By locating the valve on the calorimeter it was possible to evacuate the fill line, eliminating the superfluid heat leak below the lambda point. The heaters and GRTs were attached to the spherical wall of the calorimeter via sapphire posts which minimized stray a.c. power due to capacitive ground loops.

The internal dimensions of the calorimeter set the inner limits at which distortion of the heat capacity curve due to residual acceleration and finite size phenomena reach a given value. For a $3.5 \mathrm{~cm}$ sample and a dc acceleration level of $2 \times 10^{-4} \mathrm{~g}$, easily attained on the Shuttle, the resolution at which the pressure gradient distortion of the heat capacity reaches $1 \%$ is $\sim 3 \times 10^{-10}$. The corresponding resolution for finite size distortion is $\sim 2 \times 10^{-11}$, based on the recent renormalization group model of this effect ${ }^{11}$. Since the magnitude of the finite size effect is somewhat uncertain, it was decided to keep the calorimeter as large as possible consistent with mechanical constraints.

A charcoal adsorption pump assembly was attached to the top plate of the vacuum can to maintain a high vacuum inside the thermal control system. This pump was equipped with a heater and thermometer to allow regeneration after the experiment had reached earth orbit. During the launch phase the vacuum can was filled with ${ }^{3} \mathrm{He}$ exchange gas to maintain a thermal short between the instrument and the bath. This procedure was needed to reduce the effect of vibration heating on the HRTs. The pump-down time was on the order of a day, dependent on the vacuum can wall temperature. Residual gas in the vacuum can formed an additional thermal link between the calorimeter and its surroundings, giving rise to small thermal gradients and offsets in the calorimeter assembly. For the pressures encountered during the mission, the gradients were estimated to be less than $10^{-10} \mathrm{~K} / \mathrm{cm}$ in stage 5 .

\section{FLIGHT EXPERIMENT}

The instrument was maintained at $4 \mathrm{~K}$ or colder for 18 months before launch. In this period extensive tests were performed to verify its performance under various conditions. A number of preflight calibrations and heat capacity measurements were also completed. This data was very useful for deciding the details of the flight measurement sequence and for studying the general behavior of the system. Shortly before launch the helium in the dewar and the instrument were cooled to about $1.7 \mathrm{~K}$, and the instrument vacuum space was filled with ${ }^{3} \mathrm{He}$ exchange gas at a pressure of about $5 \mathrm{~mm}$. About two hours into the flight, the experiment was activated and the exchange gas pump-out procedure was 
started. This consisted of first venting to space until a pressure of 0.5 torr was reached, and then sealing off the instrument and using the internal cryopump. The dewar was also raised to its maximum safe operating temperature in space, close to $2.05 \mathrm{~K}$, to aid in releasing adsorbed helium from the vacuum can walls.

When the pressure approached the $10^{-9}$ torr range the main measurement sequence was started. The first event of significance in the experiment was the generation of a small bubble in the calorimeter, used to establish a thermodynamic path close to constant pressure for the heat capacity measurements. This event occurred as the calorimeter warmed through $2.079 \mathrm{~K}$ and was detected by observing the small sudden cooling generated by the first order phase transition. The output from one of the GRTs on the calorimeter during this phase is shown in figure 4. The data are plotted as deviations from a best fit straight line to the temperature vs time signal. The size and polarity of the step are consistent with the known thermodynamic parameters of helium, and the temperature of the step is similar to that seen before the flight in ground testing.

Before the system could be used for high resolution work, the various stages needed to be brought to their normal operating temperatures. These temperatures were kept as close as practical to that of the calorimeter. A typical temperature profile of the thermal control system consisted of stage 5 at a given operating temperature and stage 4 in equilibrium with it, and stages 1,2 and 3 servoed at 30 , 10 and $1 \mathrm{mK}$ below stage 4 . The initial thermal alignment was performed with the calorimeter at the starting temperature for the HRT calibration sequence. Up to this point stage 4 was operated in the coarse mode, but after the thermal transients had settled, and the fine mode was activated. The final step was to reduce the drift rate of the calorimeter temperature to an acceptable value. It was found possible to reduce the drift rate of stage 5 to $\sim 10^{-12} \mathrm{~K} / \mathrm{s}$ with little difficulty.

The HRTs were then calibrated against the GRTs. This procedure consisted of a set of $1 \mathrm{mK}$ heating steps separated by 45 seconds of equilibration. The data was analyzed on the ground and verified to be within about $0.1 \%$ of the preflight results. The second phase of instrument calibration was to measure the change in the stray power dissipated in the heater circuit when it was switched on. This was done by measuring the heat capacity of the sample as a function of power dissipated in the heater at a preselected temperature well below the lambda point. Any power change that is unaccounted for in the measuring process causes an error in the calculated heat capacity. It is easy to show that this error adds a term to the heat capacity that is inversely proportional to the power. From measurements over the range $10^{-3}$ to $10^{-8} \mathrm{~W}$, the stray power was estimated to be less than $10^{-10} \mathrm{~W}$. After the calibration was completed, heat capacity measurements using the pulse technique were commenced and continued for the rest of the flight. The quantity of energy to be dissipated in the heater for each pulse was determined from a look-up table based on the distance from the lambda point. After an initial trial pulse, this distance 
was determined from each heat capacity measurement by comparing it with a model of the singularity. After the lambda point had been reached the first time, this method was terminated and the apparatus was operated manually by ground command. Figure 5 shows the preliminary heat capacity values from the initial period on a semi-logarithmic scale. For comparison the broken line shows the expected values from a model based on ground results.

When measurements at the highest resolution were attempted, excess noise was found on the outputs of the HRTs. This was traced to the heat dissipation due to cosmic rays and other particles passing through the sensitive element of the thermometers. A comparison of the HRT output on the ground and in space is shown in figure 6 . A slow variation in the drift rate of the calorimeter was also detected under nominally steady conditions. This was found to be due to charged particles dissipating energy in the copper calorimeter. To obtain accurate heat capacity results it is necessary to correct the raw data for the particle heating effects. Heating of the calorimeter by charged particles was anticipated, so the experiment carried a charged particle monitor for use as an indicator of periods of severe heating. The variation of the charged particle counting rate over a $24 \mathrm{hr}$. period is shown in figure 7 . The four channels of data correspond to different energy thresholds for the particles. The background level of from 2 to 5 counts per second corresponds to the cosmic ray flux and the higher peaks are due to the particles trapped in the radiation belts. Models are being developed to correct the high resolution results for the heating effects. It is expected that a resolution of about 2 nanodegrees will be possible with good accuracy. Additional heat capacity data can be extracted from the cooling curves obtained when the calorimeter temperature is reset below the lambda point. However it is apparent that for this case careful modeling of thermal relaxation effects close to the transition will be needed. Some preliminary results in the high resolution region are shown in figure 8 . While it is too early to draw any conclusions of theoretical interest from the data, it can be seen that the transition appears to be sharp on the scale of nanodegrees, as expected. An examination of selected measurements puts a limit of no more than 2 nanodegrees on the transition width. The correlation length at this resolution is expected to be about 150 microns. For comparison, very high resolution ground measurements on a helium sample $0.4 \mathrm{~mm}$ high are shown in figure 9 . It can be seen that in this case the results are significantly perturbed by gravity and other effects over a range of about 200 nanodegrees.

Above the transition the calorimeter temperature initially overshoots its equilibrium value when a heat pulse is applied due to the finite thermal conductivity of the helium. From the resulting relaxation data an estimate of the thermal conductivity can be obtained. A typical response curve to a five second heat pulse is shown in figure 10. Also shown is a simple exponential fit to the relaxation data. The analysis of this data is less well developed than that for heat capacity, but some of the relaxation data has been converted into the equivalent solid body thermal conductivity. A comparison with expected 
values based on extrapolation of ground results is shown in figure 11. When fully analyzed we expect the measurements to give thermal conductivity data for helium in the normal phase to within about ten nanodegrees of the transition.

\section{ACKNOWLEDGMENTS}

We wish to thank the Microgravity office of NASA for its support with contract JPL-957448, and the members of the LPE program at Stanford and JPL for their many contributions to the program, and Ball Aerospace for construction of major portions of the flight instrument. 


\section{REFERENCES}

1. F. M. Gasparini and I. Rhee, Progr. Low Temp. Phys. ,13, 1 (1992).

2. J. A. Lipa, 75th Jubilee Conference on Helium-4, ed. J. G. M. Armitage (World Scientific, Singapore, 1983), p. 208.

3. K. G. Wilson, Phys. Rev. B 4, 3174 (1971).

4. J. A. Lipa and T. C. P. Chui, Phys. Rev. Lett. , 51, 2291 (1983).

5. D. Petrac, U. Israelsson and T. Luchik, Cryogenics, (1993), to be published.

6. J. A. Lipa, B. C. Leslie and T. C. Wallstrom, Physica ,107 B, 331 (1981); T. C. P. Chui and J. A. Lipa, Proc. 17th Internat. Conf. on Low Temp. Phys. (Karlsruhe, 1984), p.931; M. J. Adriaans, T. C. P. Chui, M. Ndesandjo, D. R. Swanson and J. A. Lipa, Physica, B 169, 455 (1991); and T. C. P. Chui, D. R. Swanson, M. J. Adriaans, J. A. Nissen, and J. A. Lipa, Temperature, its Measurement and Control in Science and Industry, 6, 1213 (1992).

7. D. Marek, Jap. Journal App. Phys., 26, Suppl. 26-3, p. BO08 (1987).

8. T. C. P. Chui, D. Swanson, M. J. Adriaans, J. A. Nissen, and J. A. Lipa, Phys. Rev. Lett., 69, 3005 (1992).

9. J. A. Lipa, T. C. P. Chui, J. A. Nissen and D. R. Swanson, Temperature, its Measurement and Control in Science and Industry, 6, 949 (1992).

10. Nippon Mining Co., Japan, grade 6N (99.9999\% pure).

11. R. Schmolke, A. Wacker, V. Dohm, and D. Frank, Physica, B $165 \& 166,575,(1990)$. 


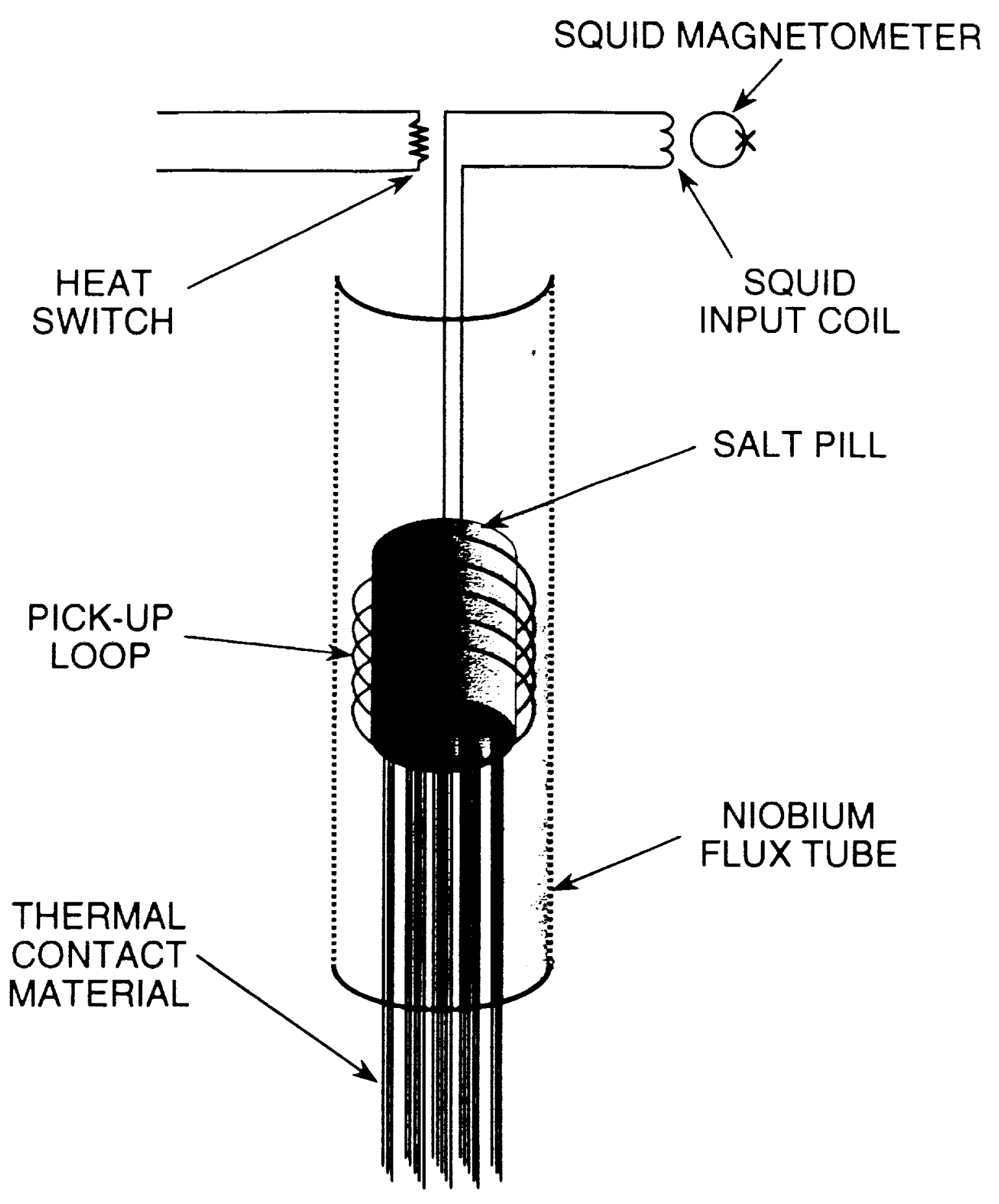

\section{PARAMAGNETIC SALT THERMOMETER}

Figure 1 Schematic diagram of the high resolution thermometers. 


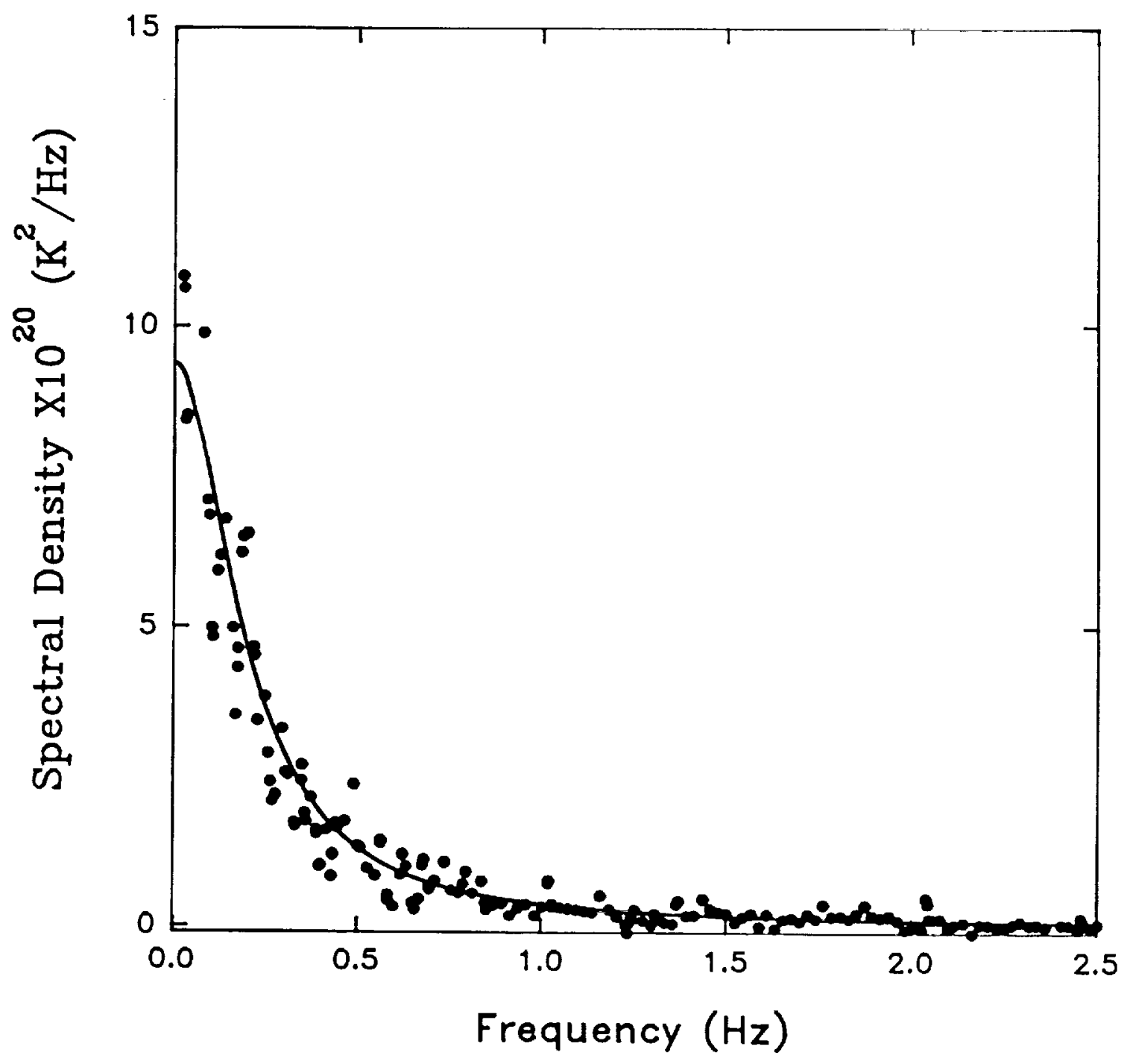

Figure 2 The noise spectrum of a high resolution thermometer. The solid line is the prediction of the fluctuation-dissipation theorem with no adjustable parameters. 


\section{LAMBDA-POINT EXPERIMENT FLIGHT INSTRUMENT}

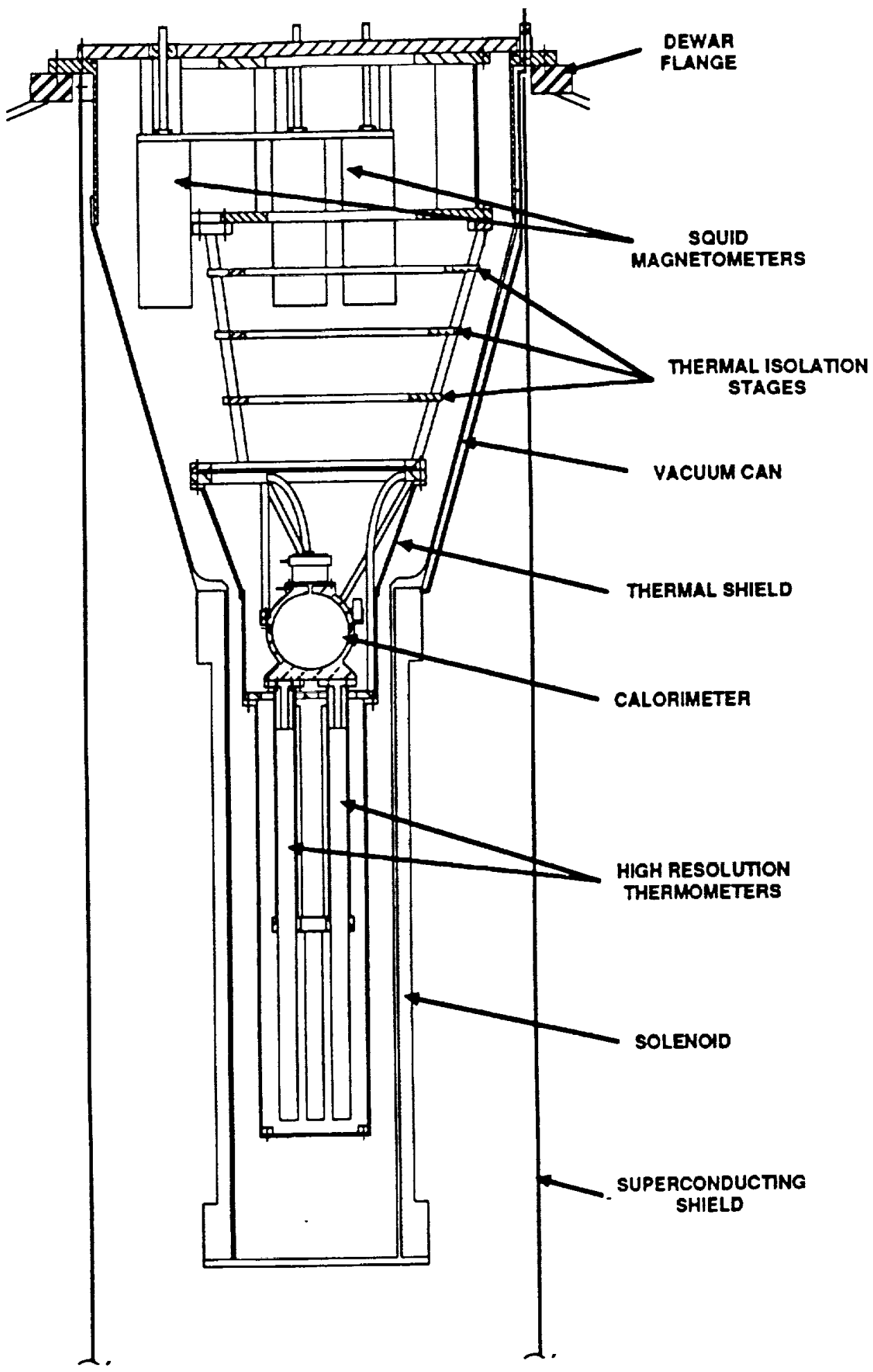

Figure 3 Schematic view of the thermal control system and calorimeter. 


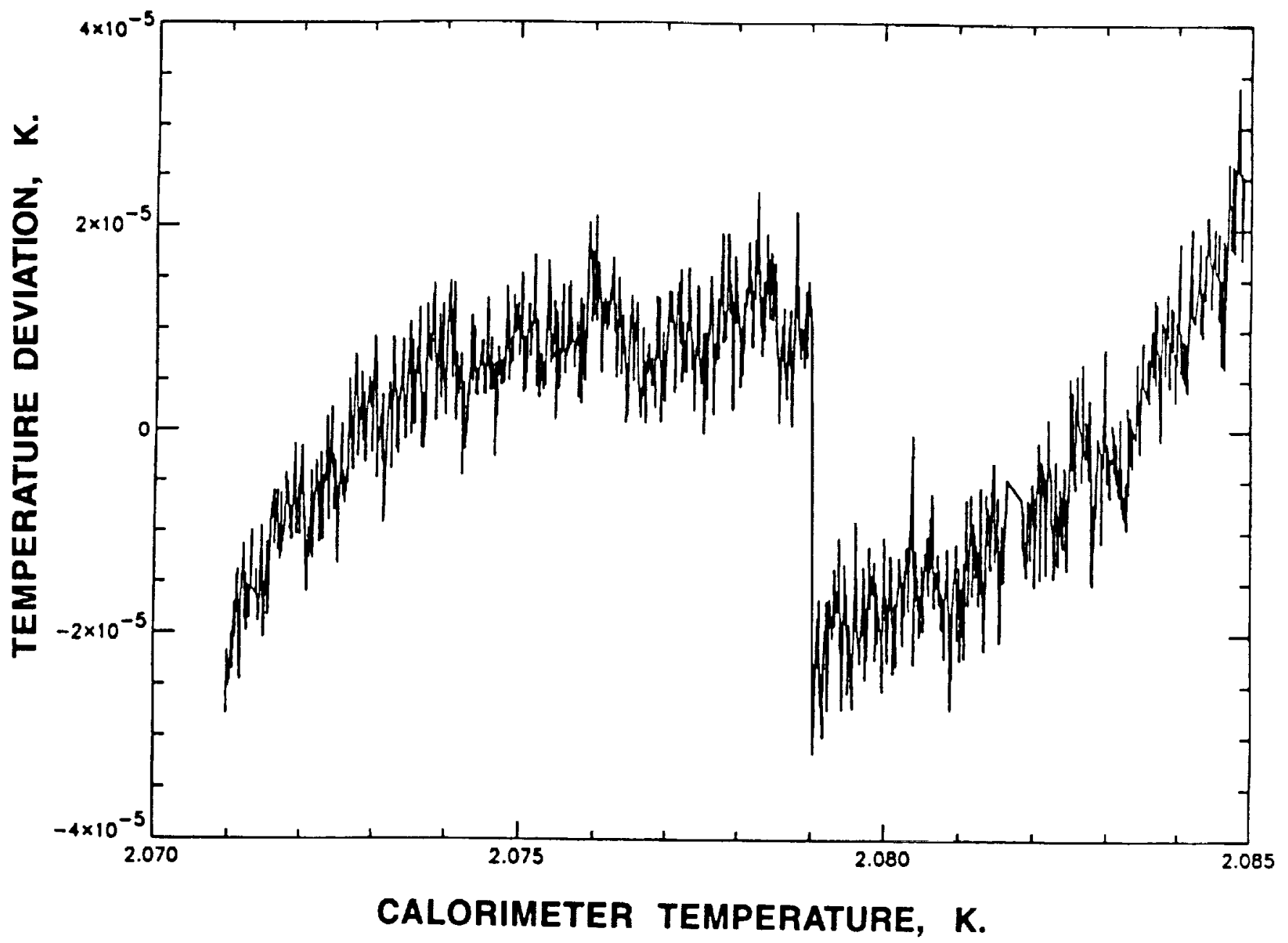

Figure 4 Output of calorimeter GRT showing bubble generation event near $2.079 \mathrm{~K}$. Data is plotted as deviations from a best fit straight line to the raw signal. 


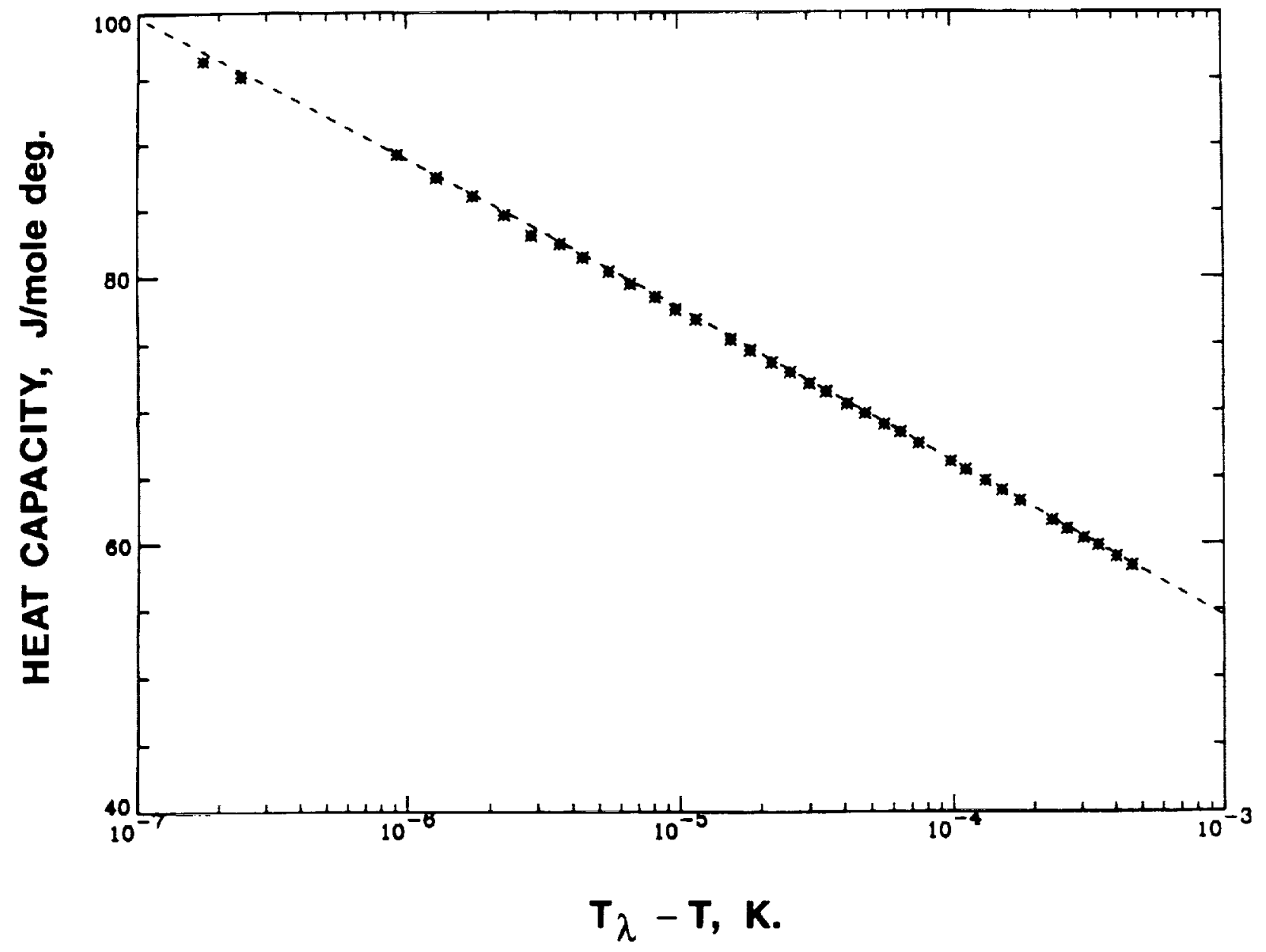

Figure 5 Wide range heat capacity data obtained early in the mission. Broken curve shows expected results based on earlier ground measurements. 


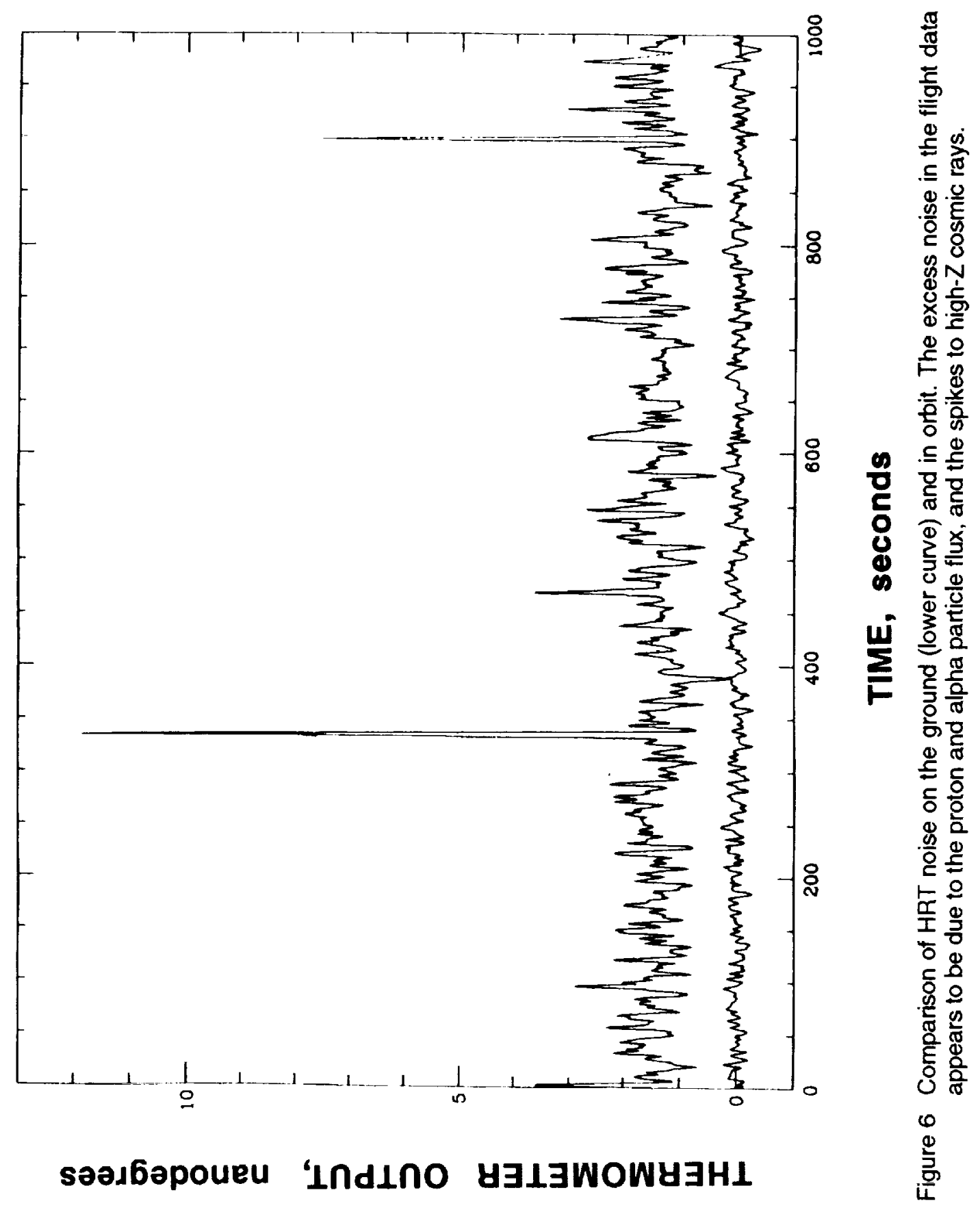




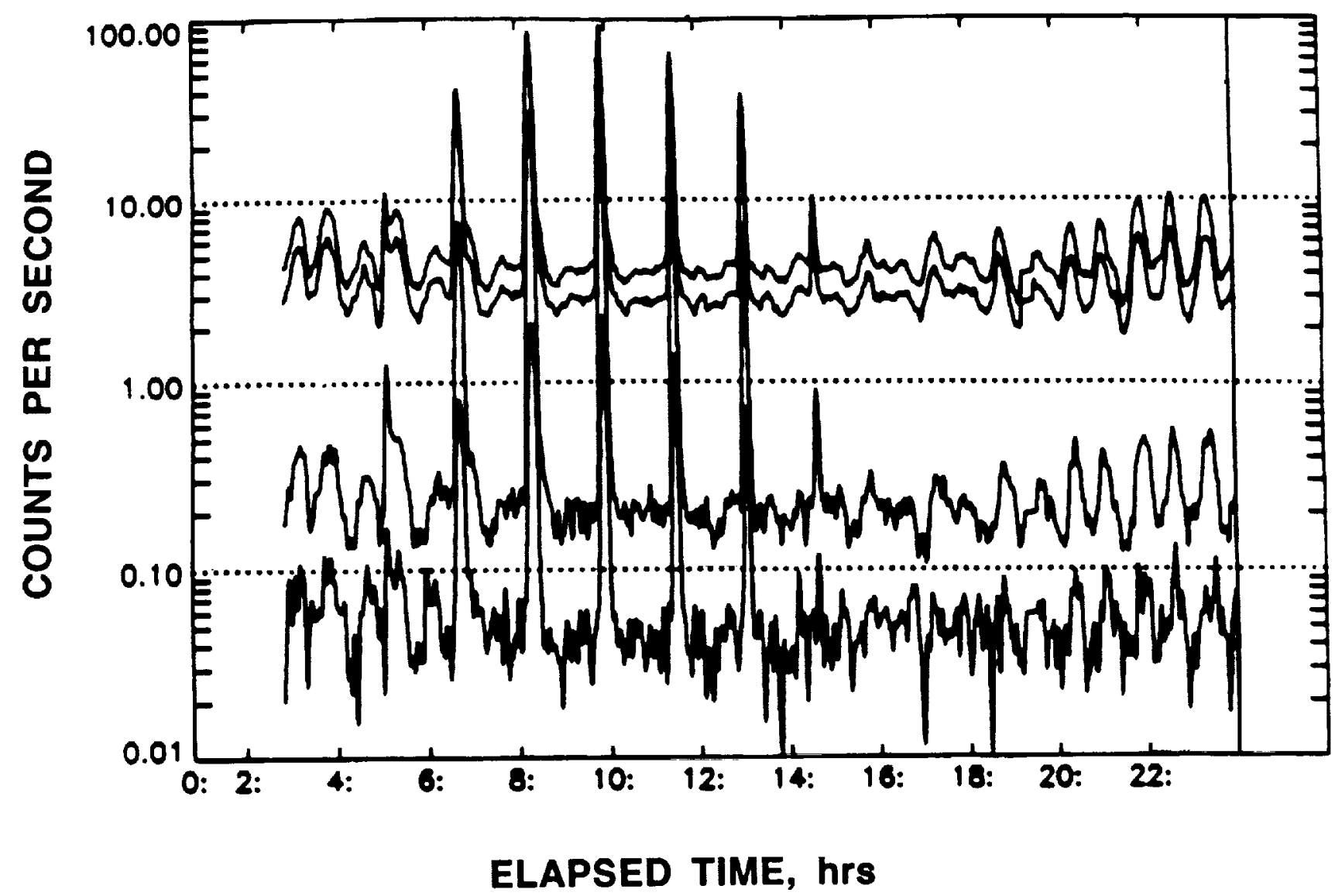

Figure 7 Variation of charged particle count rate over a $24 \mathrm{hr}$. period. Four channels correspond to different energy ranges. 


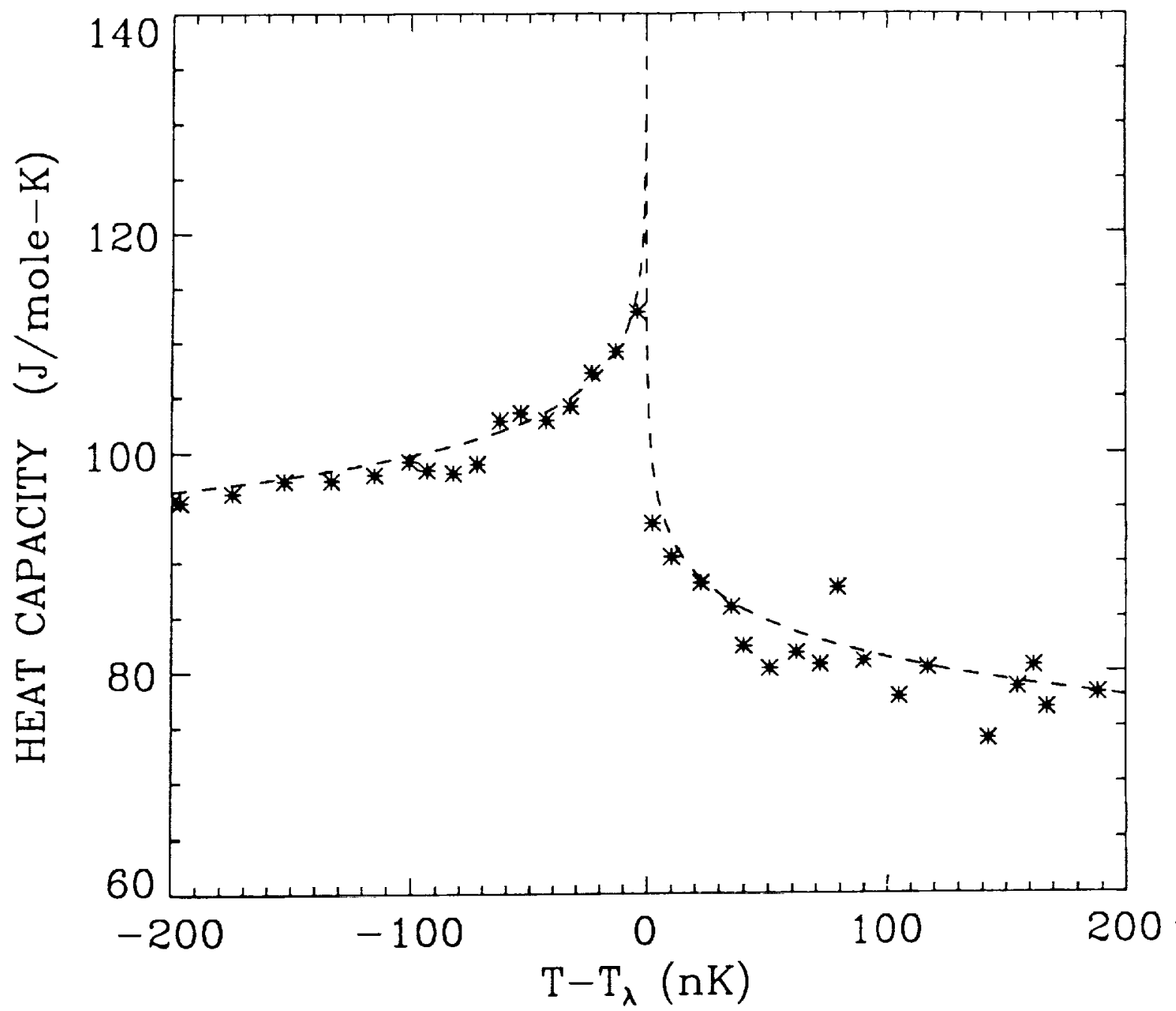

Figure 8 Preliminary high resolution heat capacity results in the region near the transition. 


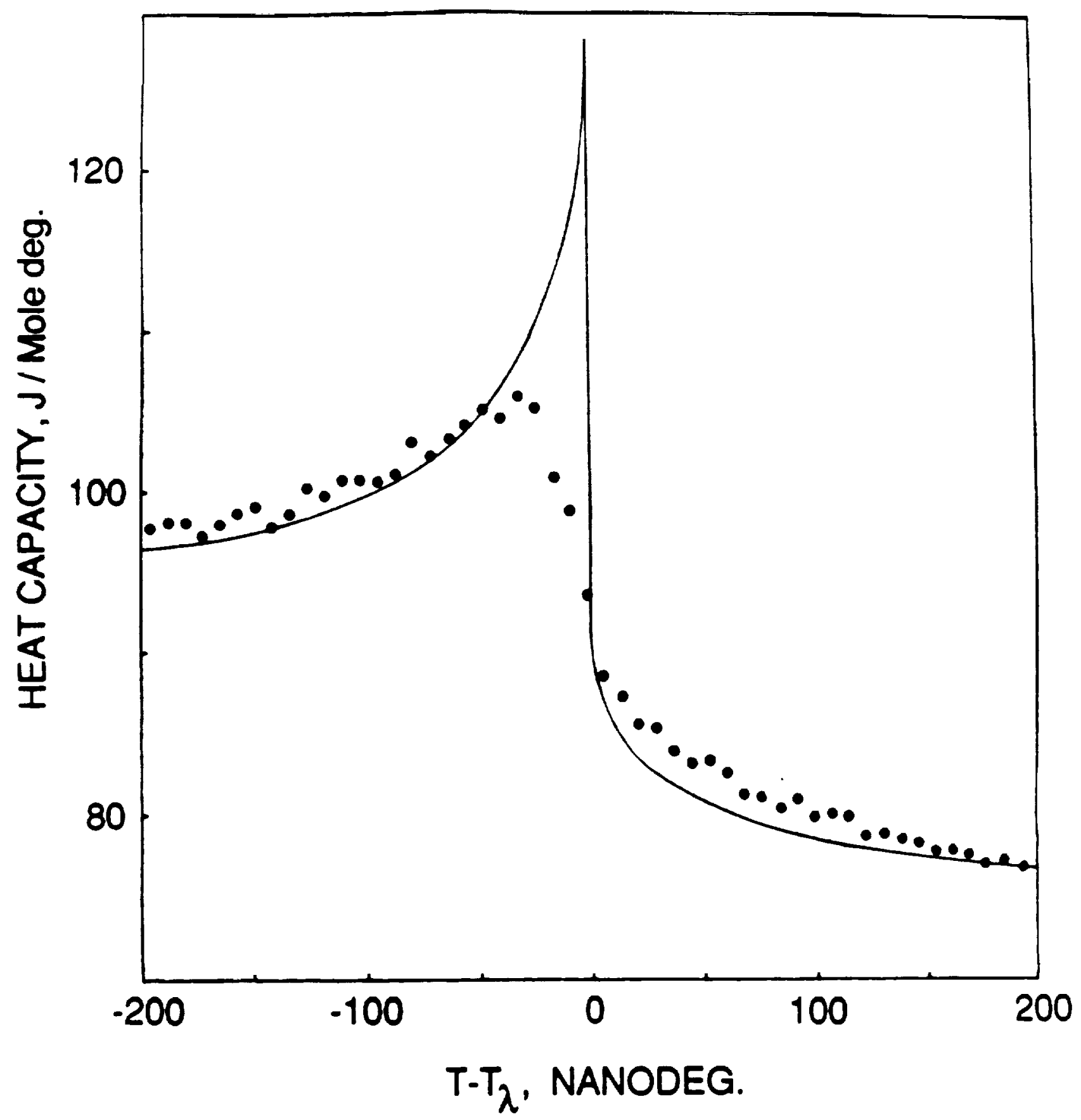

Figure 9 High resolution heat capacity measurements obtained on the ground showing the effects of gravitational and finite size distortion. Solid curve schematically shows expected curve from flight results. 


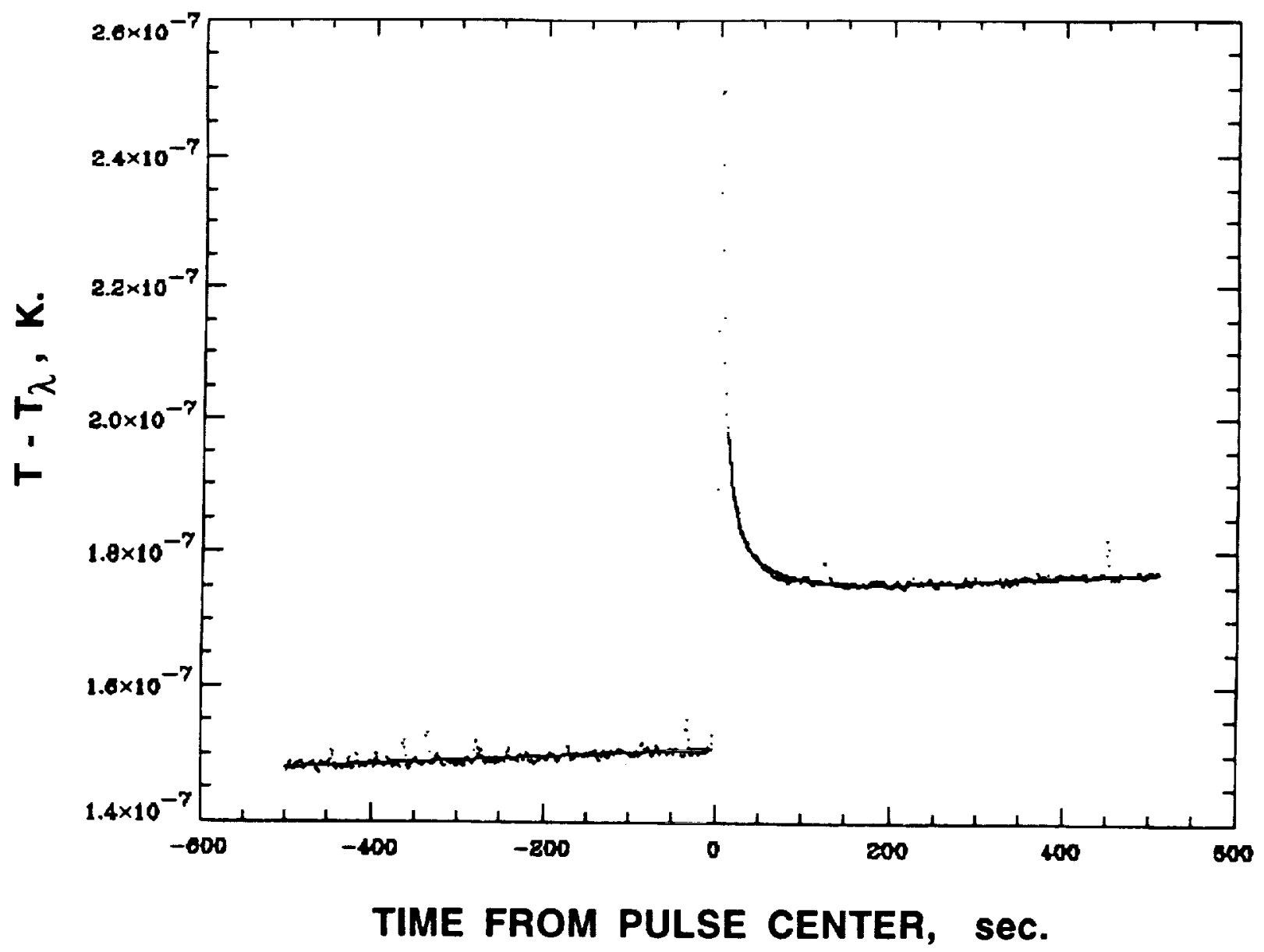

Figure 10 Temperature vs time data from a single heat pulse above the transition. Overshoot is due to the presence of normal helium in the calorimeter. Solid line is a simple exponential fitted to the high temperature data. 


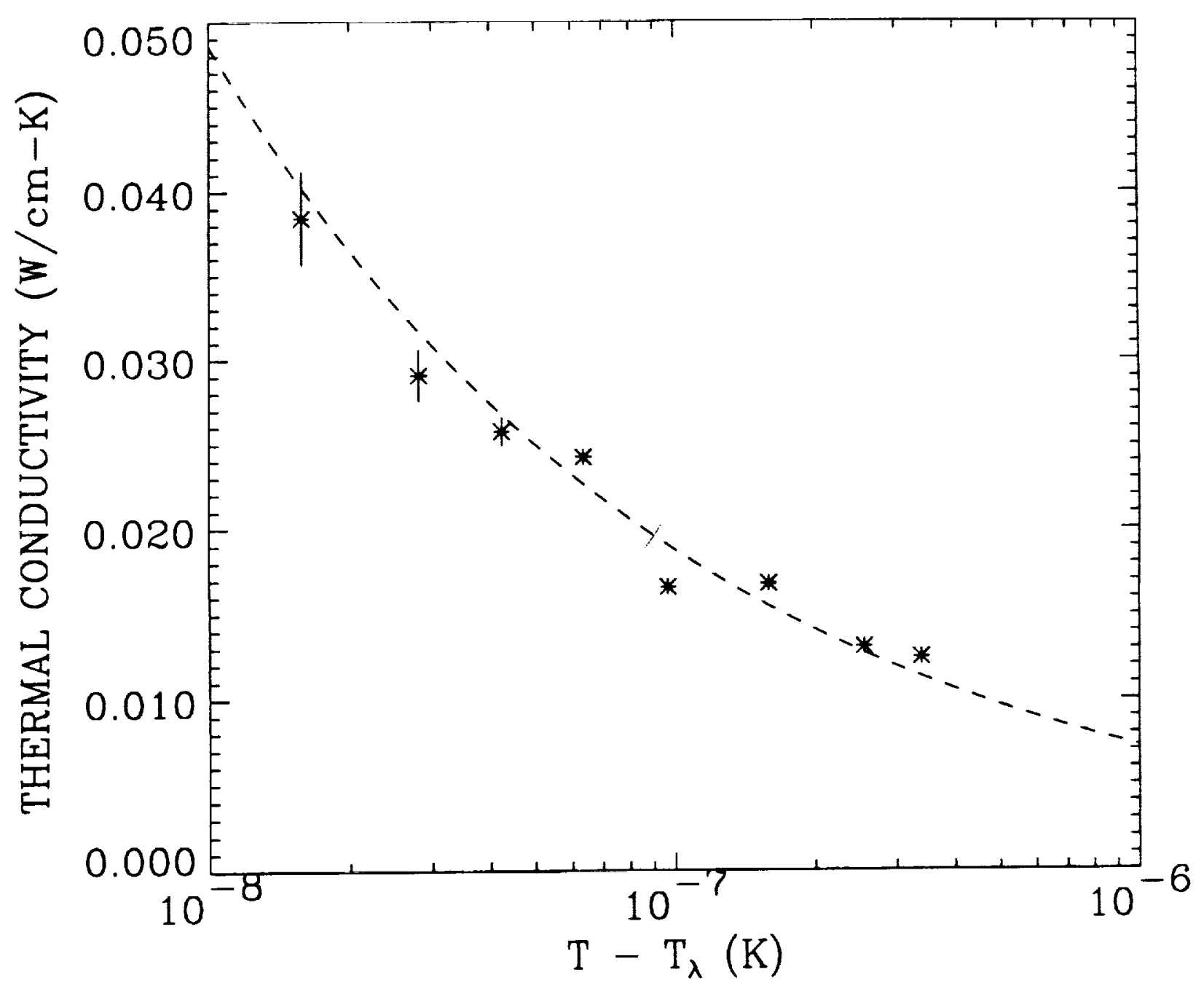

Figure 11 Preliminary comparison of expected (solid curve) and observed thermal conductivity in the region just above the transition. 


\section{Discussion}

Question: Could you comment on the effects of radiation interactions (in terms of heating the fluid) on the experiment?

Answer: There are two effects; one is heating of the fluid which is pretty negligible because the cross section for absorption of cosmic rays of the fluid is fairly low. The calorimeter that the helium is contained in has a relatively high cross section and most of the actual heating as a function of time occurs in the calorimeter. However there are also the particles that go through the salt of the thermometer itself and they do very local heating in the salt and they give you a burst of spike-like energy which you see in your output. They make noise but the total amount of heating in the salt in absolute terms is relatively small compared to the calorimeter.

Question: Following up on the last question, do you see any correlations of this effect?

Answer: Yes. You can. You see correlations in the output about $20 \%$ of the time. That corresponds approximately to the solid angle that is subtended by the other one in its field of view. There is also some indication that the particles are not just the prime reason; they are more of a shower effect, you get a few more events than you would expect than just from the solid angle argument. But a lot of the noise is uncorrelated.

Question: In your opinion, do you think that the ground based (Lambda point) data will or will not be altered by the microgravity data in terms of the disparity between the experimental and the theoretical exponent?

Answer: Well just from what I have seen over the years I would expect the flight data will be better by the time the analysis is completed than just the ground based measurement alone, but I think we will also probably have to take into account the ground based measurements and do analysis which sort of folds the two together to some extent. That is because in the outer regions far from the transition there are terms which go to zero but don't go to zero very quickly. These have to be considered and these are best determined from ground based measurements. I would think the issue of whether the discrepancy is as serious or not will depend on a couple of things. One is the uncertainties in the various experiments right now. Statistical uncertainty is very common in experimental work but the true underlying uncertainties are somewhat larger. Also the uncertainties in theoretical models are not negligible. It turns out, fortunately, that there has been a bit of a breakthrough in the last couple of years in the area of numerical modeling of phase transitions using Monte Carlo techniques and we are expecting there will be better estimates to come out quite soon on the experiments from the theoretical side. So it is a bit hard to say at this point what the final situation is going to be. I think it is still in a bit of a flux. 
